\title{
Cellular Physiology

\section{Panax notoginseng Saponins Potentiate Osteogenesis of Bone Marrow Stromal Cells by Modulating Gap Junction Intercellular Communication Activities}

\author{
Xue-dong Li ${ }^{1}$, Bo Chang ${ }^{1}$, Bin Chen ${ }^{1}$, Zhao-yong Liu', Dong-xin Liu ${ }^{1}$, \\ Jian-sheng Wang ${ }^{1}$, Guo-qing Hou ${ }^{1}$, Dong-yang Huang ${ }^{2}$ and Shi-xin \\ $\mathrm{Du}^{1}$
}

${ }^{1}$ Department of Orthopaedics, the First Affiliated Hospital, Shantou University Medical College, Shantou, ${ }^{2}$ Center for Molecular Biology, Shantou University Medical College, Shantou. The first two authors contributed equally to this study

\section{Key Words}

Panax notoginseng saponins $\cdot$ Bone marrow stromal cells - Gap junction intercellular communication • Osteogenesis

\begin{abstract}
Aims: The Chinese medicinal herb, Panax notoginseng, has long been used to treat bone fractures and Panax notoginseng saponins (PNS) could promote bone formation. We investigated the effects of PNS on gap junction intercellular communication (GJIC) and osteogenesis-associated genes in rat bone marrow stromal cells (BMSCs). Methods and Results:Our MTT assays demonstrated that PNS enhanced BMSC proliferation under basal medium culture in vitro. Alkaline phosphatase (ALP) assays and alizarin Red staining showed that PNS stimulated ALP activity and calcium deposition by BMSCs. Measurement of the traversing of Lucifer yellow through intercellular junctions revealed that PNS significantly stimulated GJIC activities. RT-PCR assays further showed that PNS augmented the increase in the mRNA levels of ALP, core-binding factor a1, and bone sialoprotein while decreasing the mRNA level of PPAR $\gamma 2$. PNS also reduced RANKL levels and
\end{abstract}

\section{KARGER}

Fax +4161306 1234

E-Mail karger@karger.ch

www.karger.com
(C) 2010 S. Karger AG, Basel

$1015-8987 / 10 / 0266-1081 \$ 26.00 / 0$

Accessible online at:

www.karger.com/cpb increased osteoprotegerin levels. Gap junction inhibitor, 18a-glycyrrhetinic acid, could partially reverse the actions of PNS on BMSCs. Conclusions: Our findings indicate that PNS could promote osteogenesis of BMSCs by targeting osteogenesisassociated genes, which could be mediated by their actions on GJIC.

Copyright @ 2010 S. Karger AG, Basel

\section{Introduction}

Bone marrow stromal cells are pluripotent cells that can be induced to undergo differentiation into multiple cell lineages including osteoblasts, chondrocytes, adipocytes, and other types of cells and they are often considered the precursor cells of the osteoblast lineage [1].These cells have been attempted for bone regeneration in animal models [2] and, when implanted in vivo on a three-dimensional scaffold into immunocompromised mice, they could form bone and hematopoiesis-supportive stroma. Ex vivo expanded bone marrow stromal cells have been employed in clinical trials for repairing large bone segmental defects [3]. 
Bone repair is a dynamic process achieved by bone remodeling that involves the coordinated actions of osteoclasts, osteoblasts, osteocytes within the bone matrix, and osteoblast-derived lining cells that cover the bone surface. Osteoblasts are at the epicenter of this process because they are responsible for matrix production and mineralization of the bone, and they also receive and process most mechanical and chemical signals to the bone, and likely direct osteoclast function [4]. Bone remodeling is initiated with osteoclast formation followed by osteoclast-mediated bone resorption and subsequent osteoblast-mediated bone matrix formation with the mineralization of the matrix. Direct intercellular contact and communication are of obvious relevance to osteoblasts, which are interconnected by abundant junction structures, including gap junctions. Gap junctions are aqueous intercellular channels that are formed by the juxtaposition of two hemichannels in adjacent cells and allow the diffusion of small molecules and ions from cell to cell. Abundant evidence indicates that gap junction intercellular communication may play important roles in transducing mechanical signals throughout the bone cells and is critically important in osteoblast proliferation and differentiation [5].

Panax notoginseng is a traditional Chinese herbal medicine and has been used to promote bone healing after fracture since ancient time. The main bioactive constituents of Panax notoginseng are Panax notoginseng saponins (PNS) [6] whose three major components are ginsenoside $\mathrm{Rg}_{1}$ and $\mathrm{Rb}_{1}$, and notoginsenoside $\mathrm{R}_{1}$ [7] Panax notoginseng saponins could produce a myriad array of pharmacological responses such as antioxidative [8] and anti-inflammatory response [9]. Ginsenoside $\mathrm{Rg}_{1}$, a major component of Panax notoginseng saponins, could stimulate alkaline phosphatase activities and increase the number of osteoblasts in vitro [10]. Additionally, ginsenoside $\mathrm{Rg}_{1}$ was found to promote the proliferation of bone marrow stromal cells [11]. In ovariectomized rats, Panax notoginseng saponins could improve bone strength and trabecular microarchitecture and promote bone mineral density [12]. It was also found that ginsenoside $\mathrm{Rg}_{1}$, when incorporated into polypropylene fumarate and calcium phosphate bone cement composite, could promote angiogenesis of osteonecrosed femoral head with disrupted bone blood supply [13]. These findings suggest that Panax notoginseng saponins could be of potential therapeutic uses for such conditions as bone nonunion, osteoporosis and osteonecrosis.
Ginsenoside has also been found to be capable of modulating gap junction intercellular communication. Ginsenoside Rg1 could protect against hypoxia/ reoxygenation-induced injury to gap junction intercellular communication [14] whereas ginsenoside-Rd could inhibit interleukin 1 alpha-mediated reduction in gap junction intercellular communication activities [15]. We hypothesized that Panax notoginseng saponins could promote osteogenesis of bone marrow stromal cells by modulating the activities of gap junction-mediated intercellular communication. In this study, we isolated rat primary bone marrow stromal cells and investigated the effects of Panax notoginseng saponins on bone marrow stromal cells under osteogenic induction. We further studied whether Panax notoginseng saponins promoted osteogenesis of bone marrow stromal cells through the involvement of gap junction-mediated intercellular communication.

\section{Materials and Methods}

\section{Preparation of bone marrow stromal cells}

Four-week-old male Sprague-Dawley rats were purchased from the Experimental Animal Center of Shantou University Medical College, Shantou, China. The animals were housed in environmentally controlled conditions $\left(22^{\circ} \mathrm{C}\right.$, a $12-\mathrm{h}$ light/ dark cycle with the light cycle from 6:00 to $18: 00$ and the dark cycle from 18:00 to 6:00) with ad libitum access to standard laboratory chow. The study protocol was approved by the local Institution Review Board and all animal experiments were performed according to the guidelines of Institutional Animal Care and Use Committee of Shantou University Medical College. Primary bone marrow stromal cells were collected from the epiphyseal regions of the femora and tibia as previously described [16] and primary cells at passage three to six were typically used for experiments.

\section{Flow cytometry analysis}

BMSCs surface markers were detected on passage three cultures of bone marrow stromal cells using flow cytometry and the following procedure. $10^{6}$ cells were incubated with either isotypic control (Biolegend, San Diego,USA) or monoclonal antibody against FITC-conjugated anti-rat CD90 (Biolegend, San Diego,USA), phycoerythrin-conjugated anti-rat CD45 (Biolegend, San Diego,USA), phycoerythrinconjugated anti-rat CD44 (Serotec, Kidlington,UK), and phycoerythrin-conjugated anti-rat CD11b (Biolegend, San Diego,USA) for $30 \mathrm{~min}$ on ice in the dark. Then the incubated cells had been washed twice with cold HBSS, surface markers were measured by flow cytometry.

Li/Chang/Chen/Liu/Liu/Wang/Hou/Huang/Du 


\begin{tabular}{|c|c|c|c|}
\hline $\begin{array}{l}\text { Gene and Genebank } \\
\text { accession no. }\end{array}$ & Primer sequence (forward/reverse) & $\operatorname{Tm}\left({ }^{\circ} \mathrm{C}\right)$ & $\begin{array}{l}\text { Product } \\
\text { size (bp) }\end{array}$ \\
\hline ALP (J03572) & 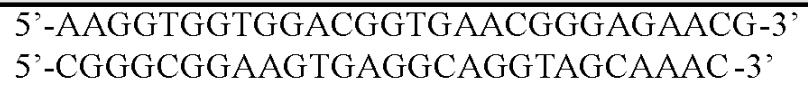 & 65.5 & 455 \\
\hline BSP (NM_012587) & $\begin{array}{l}\text { 5'-GCTATGAAGGCTACGAGGGTCAGGATTAT-3' } \\
\text { 5'-GGGTATGTTAGGGTGGTTAGCAATGGTGT-3' }\end{array}$ & 59.1 & 386 \\
\hline Cbfa1 (AF053950) & $\begin{array}{l}\text { 5'-CCTCACAAACAACCACAGAAC CA-3' } \\
\text { 5'-AACTGAAAATACAAA CCATACCC-3' }\end{array}$ & 60 & 325 \\
\hline PPAR 2 (NM_013124) & $\begin{array}{l}\text { 5'-CAGGCTTGCTGAACGTGAAG-3' } \\
\text { 5'-ACGTGCTCTGTGACAATCTGC-3' }\end{array}$ & 60 & 177 \\
\hline $\mathrm{Cx} 43(\mathrm{X} 06656)$ & $\begin{array}{l}\text { 5'-GCCTACTTCAATGGCTGCTC -3' } \\
\text { 5'-GAGTACCAACTCCACGGGAA-3' }\end{array}$ & 54.4 & 455 \\
\hline GADPH (AB017801) & $\begin{array}{l}\text { 5'-TGCTGAGTATGTCGTGGAG-3' } \\
\text { 5'-GCATCAAAGGTGGAAGAAT-3' }\end{array}$ & 52.8 & 618 \\
\hline
\end{tabular}

Table 1. PCR primer sequences and cycle conditions Note: ALP, alkaline phosphatase; BSP, bone sialoprotein; Cbfa1, core-binding factor a1; PPAR $\gamma 2$, peroxisome proliferator activated receptor gamma 2 ; $\mathrm{Cx} 43$, connexin 43; GADPH, glyceraldehyde-3-phosphate dehydrogenase.

Cell proliferation and osteogenic differentiation assays

Cells were plated in a 96 -well plate at a density of $1 \times 10^{4}$ cells per well and, after a 24-h incubation, were treated with Panax notoginseng saponins (Wu-Zhou Pharmaceutical Group, Wuzhou, China) at 10,50 , or $100 \mu \mathrm{g} / \mathrm{ml}$. Cell viability was assessed by the tetrazolium-based semi-automated colorimetric 3-(4,5-dimethylthiazol-2-yl)-2,5-diphenyltetrazolium bromide (MTT) reduction assay (Nanjing Keygen Biotech, Nanjing, China) at the appropriate time points and absorbance was read at $490 \mathrm{~nm}$ using a microtiter plate reader (KHB Labsystems Wellscan K3, Finland). For induction of osteogenic differentiation, bone marrow stromal cells were seeded at a density of $1 \times 10^{4}$ cells $/ \mathrm{cm}^{2}$ and were grown under osteogenic induction medium (OIM in Dulbecco's modified eagle medium (DMEM) (Gibco, Gaithersburg, MD) supplemented with 0.1 $\mu \mathrm{M}$ dexamethasone, $50 \mu \mathrm{M}$ ascorbate acid, and $10 \mathrm{mM} \beta$ glycerophosphate sodium). In addition, cells were treated with Panax notoginseng saponins at 10,50 , or $100 \mu \mathrm{g} / \mathrm{ml}$ in the presence or absence of $100 \mu \mathrm{g} / \mathrm{ml}$ Panax notoginseng saponins and $30 \mu \mathrm{m} 18 \alpha$-glycyrrhetinic acid $(A G A)$. Alkaline phosphatase activity was determined as previously described [17] at the appropriate time points by measuring $p$-nitrophenyl phosphate using a commercially available kit (Nanjing Jiancheng Bioengineering Institute, Nanjing, China) and was normalized against total cellular protein content. In addition, mineralization of bone marrow stromal cells was determined by alizarin Red S staining as previously described [18]. Alizarin red S concentrations were calculated by comparison with an alizarin red $\mathrm{S}$ dye standard curve and expressed as $\mathrm{nmol} / \mathrm{ml}$ after normalization against the total cellular protein and expressed as $\mathrm{nmol} / \mathrm{mg}$ protein.

Reverse transcription-polymerase chain reaction Total cellular RNA was isolated from bone marrow stromal cells using. Total RNA Kit following the manufacturer's recommended protocol (Tiangen, Beijing, China). First-strand cDNA synthesis was carried out by reverse transcription using oligo (dT) from isolated RNA samples and DNA was then used as templates for polymerase-chain reaction (PCR). The PCR

Promote Effects of Panax Notoginseng Saponins on Osteogenesis conditions and the sequences of primers are listed in Table 1 for the genes encoding the following proteins: connexin 43, alkaline phosphatase, core-binding factor a1, bone sialoprotein, peroxisome proliferator activated receptor gamma 2 (PPAR $\gamma 2$ ), and glyceraldehyde 3-phosphate dehydrogenase (GAPDH). The PCR products were resolved by $2 \%$ agarose gel electrophoresis and visualized with ethidium bromide. The relative expression of each gene was quantified by densitometry using the Gel Image Ver. 3.74 System (Tianon, Shanghai, China) and normalized against $G A P D H$.

\section{Western blotting studies}

The cells were lysed using RIPA lysis buffer containing $50 \mathrm{mM}$ Tris- $\mathrm{HCl}$ (pH 7.6), $150 \mathrm{mM} \mathrm{NaCl}, 1 \%$ sodium deoxycholate, $1 \%$ NP-40, $0.5 \mathrm{mM}$ phenylmethylsulfonyl fluoride, and $1 \mathrm{mM}$ EDTA. Supernatants were collected and protein concentration was determined by BCA assay kit. For western blot analysis, $20 \mu \mathrm{g}$ of each protein sample underwent $10 \%$ SDS-PAGE and electrotransferred onto polyvinylidene fluoride membranes. After being washed three times with PBS buffer, the membranes were blocked in 5\% nonfat dry milk for 1 $\mathrm{h}$ and incubated sequentially with primary and secondary antibodies. The primary antibodies was $\mathrm{Cx} 43$ at 1:200(Boster Biological Technology). After incubation with horseradish peroxidase conjugated secondary antibody for $1 \mathrm{~h}$ at room temperature, the immune complexes were visualized by enhanced chemiluminescence methods. The band intensity was measured and quantitfied and normalized against GADPH and analyzed using the Quantity One software (BioRad, USA).

\section{Measurement of gap junction-mediated intercellular communication and enzyme linked immunosorbent assays}

Gap junction intercellular communication activity was measured by scrape loading/dye transfer assay as described previously [19]. The number of cell layers traversed by Lucifer yellow in each well was counted in five separate fields under the microscope. For enzyme linked immunosorbent assays (ELISA), the content of the receptor activator of nuclear factor 
Fig. 1. Surface markers of BMSCs examined by flow cytometry. Establishing gates for immunophenotyping by flow cytometry (A); BMSCs were labeled with monoclonal antibodies against CD90 and CD44 (B and C), CD45 and $\mathrm{CD} 11 \mathrm{~b}$ (D and E); Positive control for CD90 (F); Positive control for CD44 and CD45 (G); Positive control for CD11b (H). Results show that the cultured cells were positive for BMSCs markers (CD90 and CD44) and negative for hematopoietic markers (CD45 and CD11b).

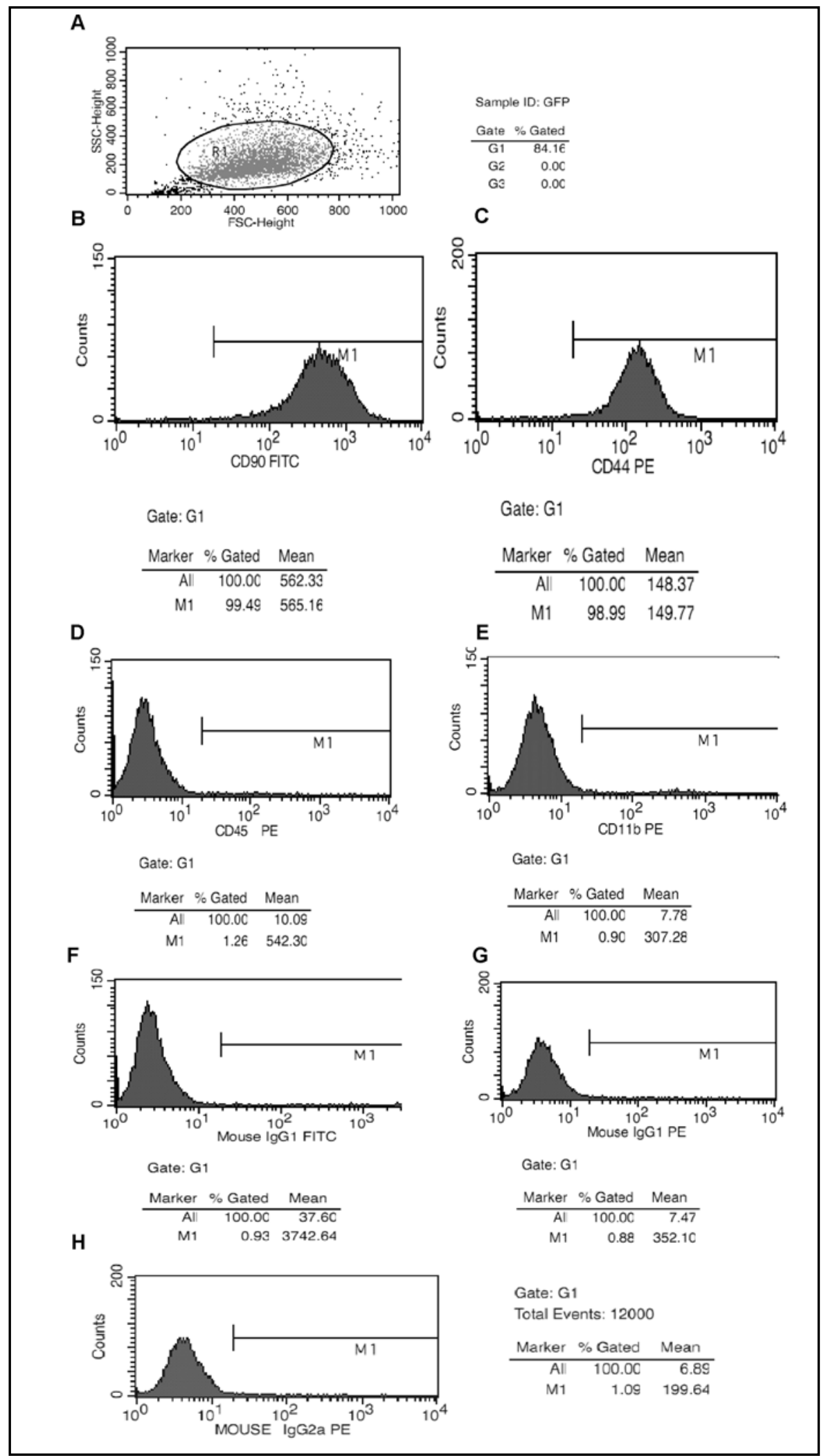

B ligand (RANKL) and osteoprotegerin(OPG) in the cell culture medium and cell lysates were measured by commercially available ELISA kits according to the manufacturer's instructions (Boster Biological Technology). RANKL and osteoprotegerin content was normalized against total cellular protein and expressed as $\mathrm{ng} / \mathrm{mg}$ protein.
Statistical analysis

The data were expressed as means \pm standard deviation (s.d.) for three or more independent experiments. Statistical significance was estimated by one-way ANOVA with Bonferroni's post test (multiple comparisons), and StudentNewman-Keuls test (comparisons between two groups) was

Li/Chang/Chen/Liu/Liu/Wang/Hou/Huang/Du 
Fig. 2. A. Panax Notoginseng saponins (PNS) promoted the proliferation of bone marrow stromal cells (BMSCs) in a dosedependent manner. BMSCs were treated with PNS at 10,50 or $100 \mu \mathrm{g} / \mathrm{ml}$ for 1 to $9 \mathrm{~d}$, and the proliferation rate was assessed by the MTT assays. The data represents the mean $\pm \mathrm{SD}$ in five times independent experiments ( $\mathrm{n}=3)$. B, C. Panax Notoginseng saponins (PNS) potentiated the mineralization of bone marrow stromal cells (BMSCs) under osteogenic induction. Mineralization was quantified by Alizarin Red S assay after BMSCs underwent osteogenic induction in the absence or presence of PNS (10, 50 and $100 \mu \mathrm{g} / \mathrm{ml})$ for 21 d. B. Alizarin Red S showed that PNS enhanced positive staining of BMSCs in a dose-dependent manner. C. The mineralization was quantified by a destaining procedure. Data represents the mean \pm SD in five times independent experiments $(\mathrm{n}=3)$. ${ }^{a} P<0.01$ versus osteogenic induction only group; ${ }^{b} P<0.01$ versus $10 \mu \mathrm{g} / \mathrm{ml}$ group; ${ }^{\mathrm{c}} P<0.01$ versus $50 \mu \mathrm{g} / \mathrm{ml}$ group. D. The effect of Panax Notoginseng saponins (PNS) on alkaline phosphatase activity of bone marrow stromal cells (BMSCs). BMSCs were cultured in basal medium, osteogenic induction medium (OIM) or OIM with 10, 50 and $100 \mu \mathrm{g} / \mathrm{ml}$ PNS for $0,3,7,11$, and $14 \mathrm{~d}$. Alkaline phosphatase (ALP) activity of the BMSCs cultured in OIM increased significantly over time, which was further significantly enhanced by PNS in OIM in a dose-dependent manner. Data represent the mean $\pm \mathrm{SD}$ in four times independent experiments $(\mathrm{n}=5) .{ }^{\text {a }} P<0.01$ versus control, ${ }^{\mathrm{b}} P<0.01$ versus OIM group; ${ }^{\mathrm{c}} P<0.01$ versus $10 \mu \mathrm{g} / \mathrm{ml}$ PNS group; ${ }^{\mathrm{d}} P<0.01$ versus $50 \mu \mathrm{g} /$ $\mathrm{ml}$ PNS group.
A

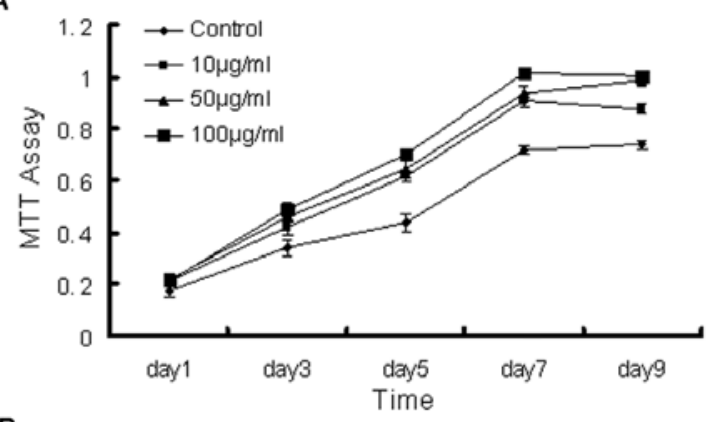

B

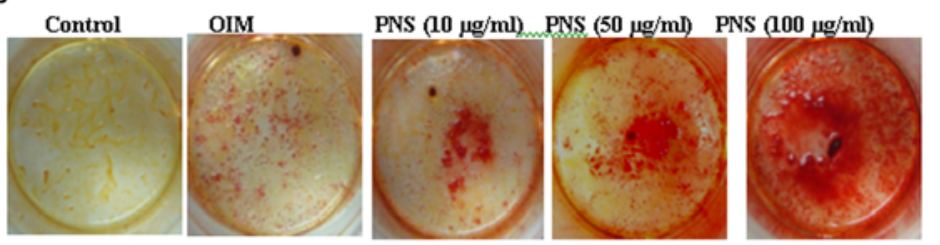

C
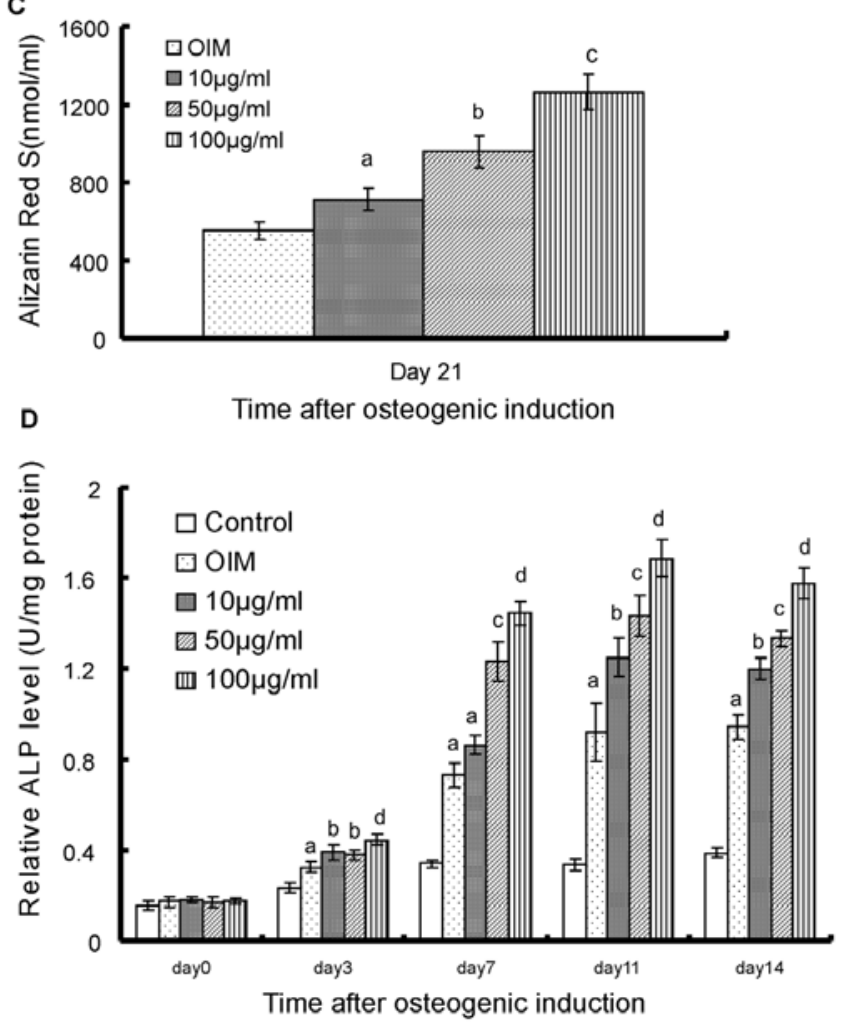

used where appropriate. A $P$ value less than 0.05 was considered statistically significant. All bioassay were at least three times independently experiments.

\section{Results}

Characterisation and phenotyping of bone marrow stromal cells

There is no single specific marker for bone marrow stromal cells. Therefore, the three passaged bone marrow stromal cells were incubated with antibodies against of

Promote Effects of Panax Notoginseng Saponins on Osteogenesis
CD90, CD44, CD45 and CD11b, and results showed that they were positive for BMSCs markers (CD90,99.49\%; CD44,98.99\%) (Fig. 1B and 1C) and negative for hematopoietic markers (CD45,1.26\%; CD11b,0.90\%) (Fig.1D and 1E).

Panax notoginseng saponins potentiate the proliferation and osteogenesis of bone marrow stromal cells

We isolated primary bone marrow stromal cells from the epiphyseal regions of the femora and tibia of rats and 
Fig. 3. Effects of Panax Notoginseng saponins (PNS) on gap junction intercellular communication (GJIC) activity and expression of connexin $43(\mathrm{Cx} 43)$. BMSCs were cultured in basal medium (Control), osteogenic induction medium (OIM), and OIM with $100 \mu \mathrm{g} / \mathrm{ml}$ PNS in the presence or absence of $18 \alpha$-glycyrrhetinic acid (AGA) for 14 days. A. The cells were subjected to scrape loading/dye transfer assay as described in 'Materials and Methods.' B. The number of dye transferred cell layers was counted at five different microscopic areas. Data are expressed as mean \pm $\mathrm{SD}$ in five times independent experiments $(n=3) .{ }^{a} P<0.01$ versus control; ${ }^{\mathrm{b}} P<0.01$ versus OIM;

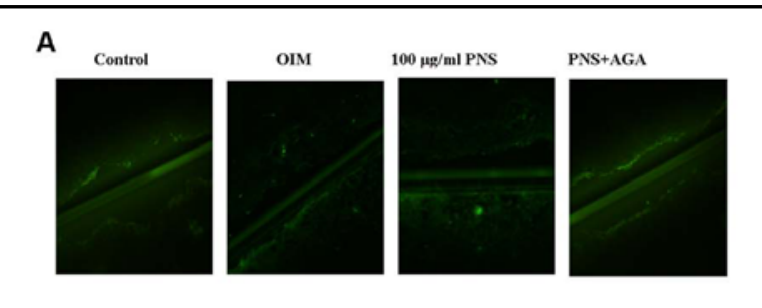

C

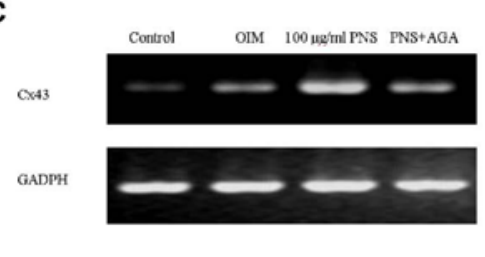

F
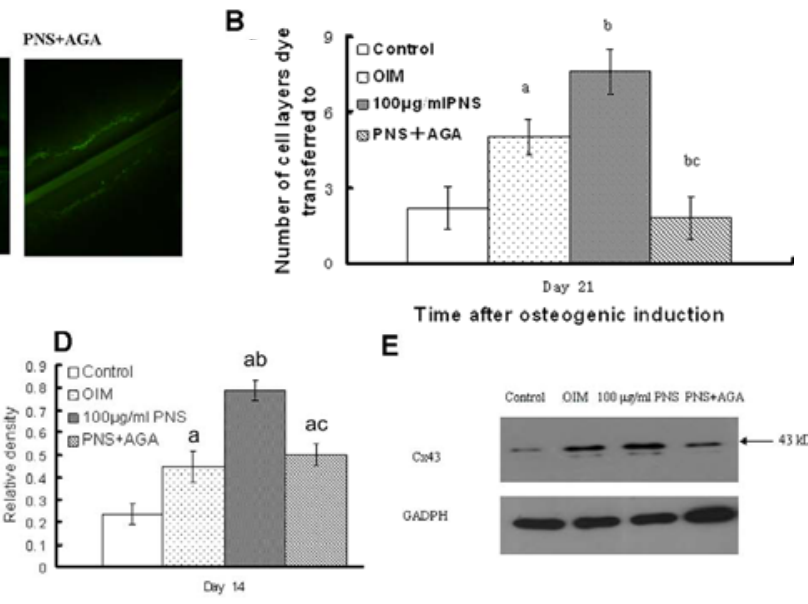

E

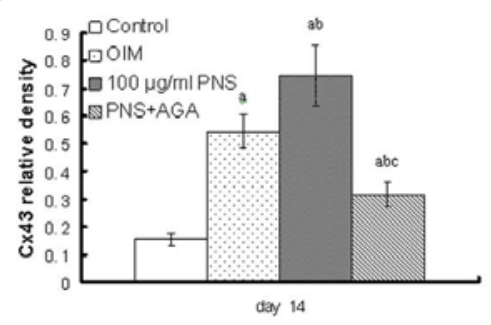

H
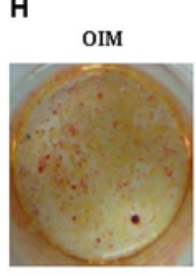

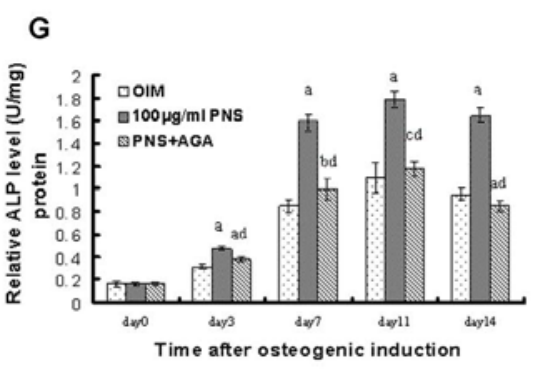

$100 \mu \mathrm{g} / \mathrm{ml}$ PNS

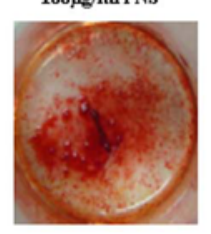

PNS+AGA

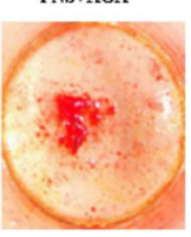

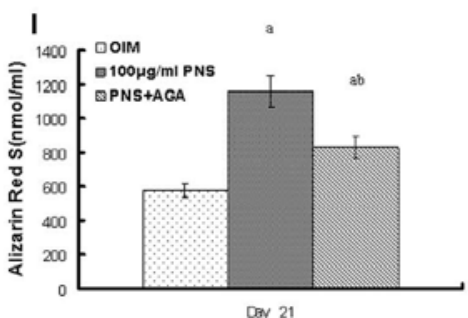

${ }^{c} P<0.01$ versus $100 \mu \mathrm{g} / \mathrm{ml}$ PNS group. C. Cx43 mRNA level were measured by RT-PCR. D. Densitometric analysis of the genes described in C. Data represents the mean $\pm \mathrm{SD}$ in three times independent experiments. ${ }^{\mathrm{a}} P<0.01$ versus control $;{ }^{\mathrm{b}} P<0.01$ versus OIM; ${ }^{\mathrm{c}} P<0.01$ versus $100 \mu \mathrm{g} / \mathrm{ml}$ PNS group. E. Western blotting analysis of $\mathrm{Cx} 43$. F. The band density in Western blotting was quantified by densitometry. Data represents the mean \pm SD in three times independent experiments. ${ }^{\mathrm{a}} P<0.01$ versus control; ${ }^{\mathrm{b}} P$ $<0.01$ versus OIM group; ${ }^{c} P<0.01$ versus $100 \mu \mathrm{g} / \mathrm{ml}$ PNS group. G. BMSCs were culture in OIM or OIM with $100 \mu \mathrm{g} / \mathrm{ml}$ PNS in presence or absence $A G A$ for $0,3,7,11$, and 14 days, then subjected to the intracellular ALP enzyme assay. Data represent the mean $\pm \mathrm{SD}$ in four times independent experiments $(\mathrm{n}=5)$. ${ }^{\mathrm{a}} P<0.01$ versus OIM $;{ }^{\mathrm{b}} P<0.05$ versus $\mathrm{OIM}$; ${ }^{\mathrm{c}} P>0.05$ versus OIM; ${ }^{\mathrm{d}} P<0.01$ versus $100 \mu \mathrm{g} / \mathrm{ml}$ PNS group. H, I. H.Mineralization was quantified by Alizarin Red S assay after BMSCs were cultured in OIM or OIM with $100 \mu \mathrm{g} / \mathrm{ml}$ PNS in the presence or absence of $A G A$ for $21 \mathrm{~d}$. Alizarin Red S showed that PNS-enhanced positive staining was attenuated by $A G A$.I. Mineralization was quantified by a destaining procedure. Data represents the mean $\pm \mathrm{SD}$ in five times independent experiments ( $\mathrm{n}=3$ ). ${ }^{\mathrm{a}} P<0.01$ versus OIM $;{ }^{\mathrm{b}} P<0.01$ versus $100 \mu \mathrm{g} / \mathrm{ml}$ PNS group.

grew them under osteogenic conditions. We then examined the effect of Panax notoginseng saponins on the growth of bone marrow stromal cells. Cell viability assays using MTT indicated that Panax notoginseng saponins dose-dependently potentiated the proliferation of bone marrow stromal cells in vitro (Fig. 2A). We examined the mineralization of these bone marrow stromal cells by alizarin Red S staining on day 21 of osteogenic induction, which revealed that, compared with the cells grown under non-osteogenic conditions, bone marrow stromal cells under osteogenic induction showed apparent calcium deposition and also exhibited increased alkaline phosphatase activities over time (Fig. 2B to 2D). We further investigated the effect of Panax notoginseng

Li/Chang/Chen/Liu/Liu/Wang/Hou/Huang/Du 
Fig. 4. A. Osteogenic and adipogenic marker gene expression was measured by semiquantitative RT-PCR. Bone marrow stromal cells (BMSCs) were grown in the osteogenic induction medium (OIM) or OIM with $100 \mu \mathrm{g} / \mathrm{ml}$ Panax Notoginseng saponins (PNS). B. Densitometric analysis of the genes described in A. Data represents the mean $\pm \mathrm{SD}$ in three times independent experiments. ${ }^{\mathrm{a}} P<0.01$ versus OIM. C, D.Osteogenic and adipogenic marker gene expression was

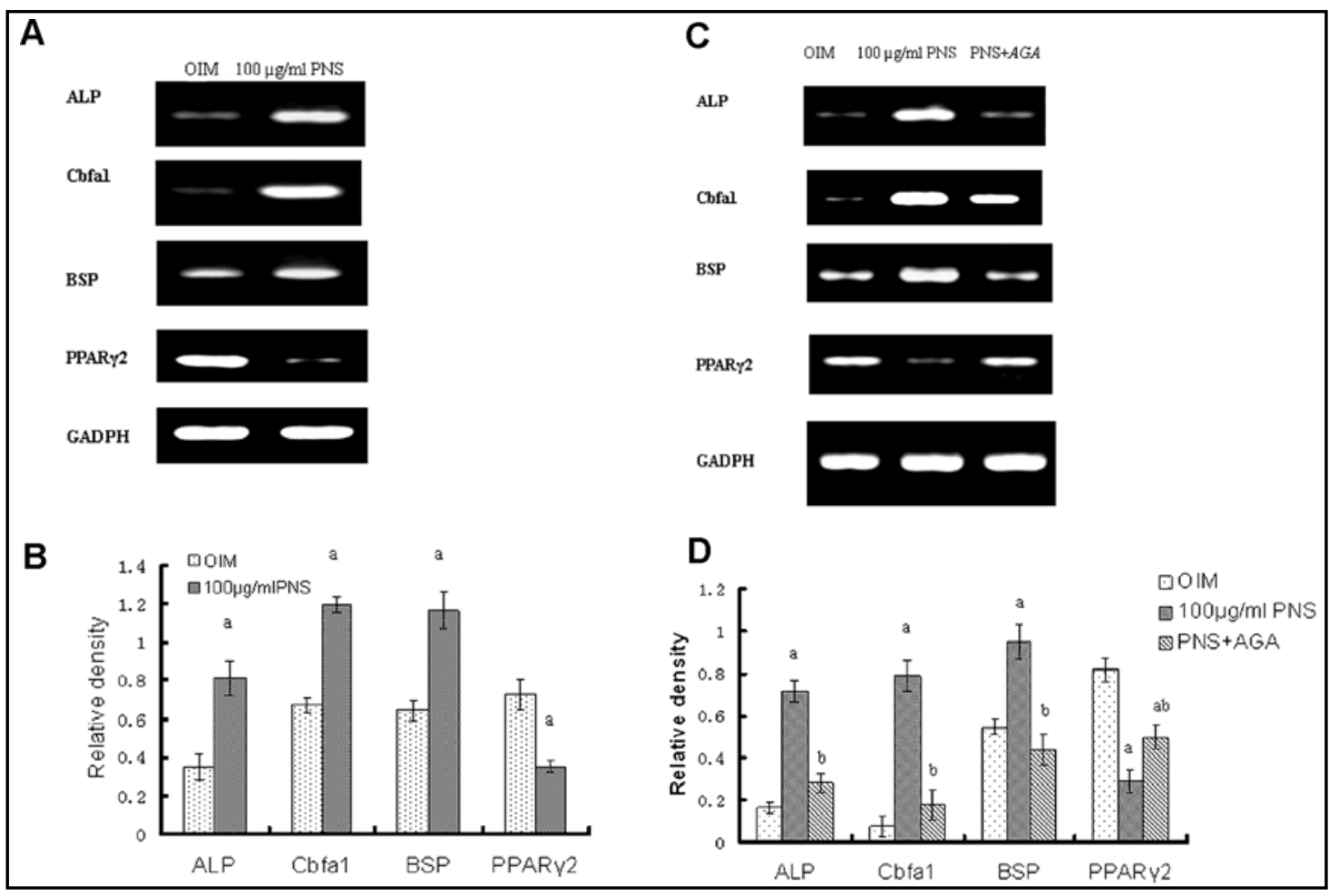

measured by semiquantitative RT-PCR. BMSCs were grown in OIM or OIM with $100 \mu \mathrm{g} / \mathrm{ml}$ PNS in the presence or absence of $A G A$ for 14 d.D. Densitometric analysis of the genes described in C. Data represents the mean \pm SD in three independent experiments. ${ }^{\mathrm{a}} P<0.01$ versus OIM ; $P<0.01$ versus $100 \mu \mathrm{g} / \mathrm{ml}$ PNS group.

saponins on the osteogenesis of bone marrow stromal cells and found that Panax notoginseng saponins promoted the mineralization of bone marrow stromal cells in a dose-dependent manner as revealed by alizarin Red $\mathrm{S}$ staining (Fig. 2B and 2C). Compared with bone marrow stromal cells under osteogenic induction only, bone marrow stromal cells treated with $10 \mu \mathrm{g} / \mathrm{ml}$ Panax notoginseng saponins exhibited a significant increase in the level of mineralization $(P<0.01)$ (Fig. $2 \mathrm{C}), 50 \mu \mathrm{g} / \mathrm{ml}$ Panax notoginseng saponins resulted in a noticeably higher mineral content in bone marrow stromal cells than those treated with $10 \mu \mathrm{g} / \mathrm{ml}$ Panax notoginseng saponins $(P<0.01)$.Furthermore, $100 \mu \mathrm{g} / \mathrm{ml}$ Panax notoginseng saponins showed the highest calcium deposit, suggesting a dose-dependent effect of Panax notoginseng saponins in stimulating the mineralization of bone marrow stromal cells. Measurement of alkaline phosphatase activities indicated that Panax notoginseng saponins caused a similar dose-dependent increase in alkaline phosphatase activities in bone marrow stromal cells under osteogenic induction (Fig. 2D). These findings together indicated that Panax notoginseng saponins could promote osteogenesis of primary bone marrow stromal cells in vitro. As 100

Promote Effects of Panax Notoginseng Saponins on Osteogenesis $\mu \mathrm{g} / \mathrm{ml}$ Panax notoginseng saponins showed the most significant differences on osteogenesis of primary bone marrow stromal cells (Fig.2C and D). Therefore, $100 \mu \mathrm{g} /$ $\mathrm{ml}$ was selected as the optimal concentration of Panax notoginseng saponins to use in the rest of the experiments.

\section{Panax notoginseng saponins potentiate} osteogenesis of bone marrow stromal cells by modulating gap junction intercellular communication activity

To further examine the mechanisms whereby Panax notoginseng saponins potentiated the osteogenesis of bone marrow stromal cells, we investigated the effects of Panax notoginseng saponins on gap junction intercellular communication, which has been considered critically important for osteoblast proliferation and differentiation [20], during osteogenic induction of bone marrow stromal cells in vitro. We studied the effect of Panax notoginseng saponins on gap junction intercellular communication activity by measuring the traversing of Lucifer yellow through intercellular junctions. We found that, compared with unstimulated cells, osteogenic induction of bone marrow stromal cells was associated 
Fig. 5. A. RANKL protein levels in the cell culture supernatants and cell lysates were evaluated by commercial ELISA kits. Data represents the mean $\pm \mathrm{SD}$ in three independent experiments $(\mathrm{n}=3) .{ }^{\mathrm{a}} \mathrm{P}<0.05$ versus Control; ${ }^{\mathrm{b}} P<0.01$ versus Control; ${ }^{\mathrm{c}} P<0.05$ versus OIM; ${ }^{\mathrm{d}} P<0.05$ versus 100 $\mu \mathrm{g} / \mathrm{ml}$ PNS group. B. Osteoprotegerin (OPG) levels in the cell culture supernatants and cell lysates were evaluated by commercial ELISA kits. Data represents the mean \pm SD in three independent experiments $(\mathrm{n}=3) .{ }^{\mathrm{a}} \mathrm{P}<$ 0.05 versus control; ${ }^{\mathrm{b}} P<0.01$ versus OIM; ${ }^{\mathrm{c}} P<0.01$ versus $100 \mu \mathrm{g} / \mathrm{ml}$ PNS group. C. Ratio of RANKL/OPG synthesis by BMSCs grown in basal medium (control), OIM or OIM with $100 \mu \mathrm{g} / \mathrm{ml}$ PNS in the presence or absence of $A G A$ for $14 \mathrm{~d}$. ${ }^{a} P<0.01$ versus control; ${ }^{\mathrm{b}} P<0.05$ versus OIM; ${ }^{\mathrm{c}} P<0.05$ versus $100 \mu \mathrm{g} / \mathrm{ml}$ PNS group.

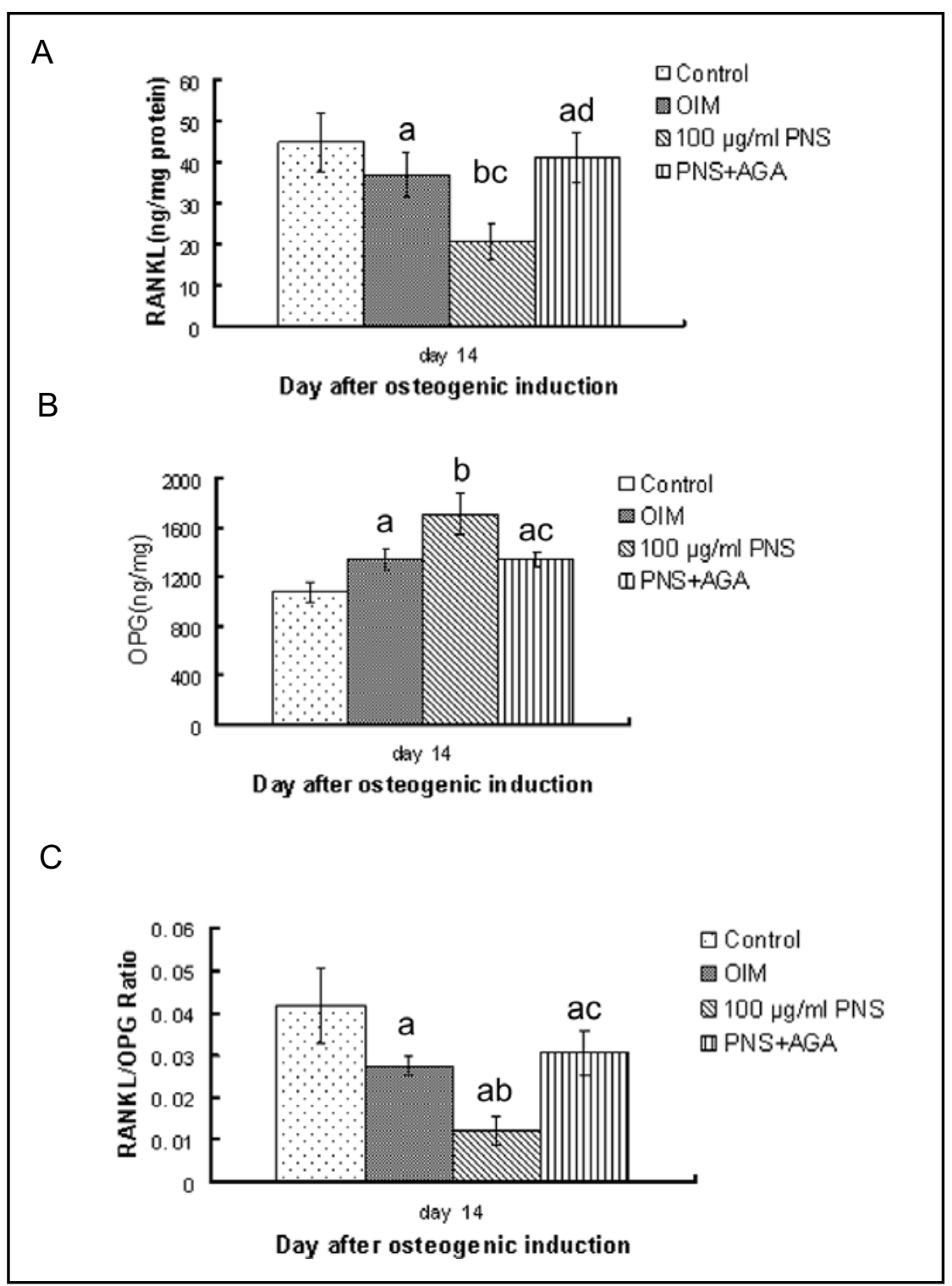

with a significant increase in gap junction intercellular communication activity at day 14 of induction $(P<0.01)$ (Fig. 3A and 3B). Additionally, $100 \mu \mathrm{g} / \mathrm{ml}$ Panax notoginseng saponins markedly potentiated the increase in gap junction intercellular communication activity in bone marrow stromal cells under osteogenic induction (Fig. 3B). Gap junction inhibitor, $18 \alpha$-glycyrrhetinic acid, could significantly attenuate Panax notoginseng saponinsmediated increase in gap junction intercellular communication activity in bone marrow stromal cells $(P$ $<0.01)$. These results indicated that gap junction intercellular communication could play a critical role in Panax notoginseng-potentiated osteogenic differentiation of BMSCs. Consistent with this role of gap junction intercellular communication, both the mRNA and protein content of connexin 43 were increased by treatment with Panax notoginseng saponins (Fig. 3C to $3 F)$. Pretreatment with $18 \alpha$ glycyrrhetinic acid, however, could attenuate the saponins-potentiated increase in connexin 43. Our findings suggested that connexin 43 could be involved in Panax notoginseng saponinspotentiated increase in gap junction intercellular communication activities in bone marrow stromal cells during osteogenic differentiation.

To further confirm that Panax notoginseng saponins could promote the osteogenesis of bone marrow stromal cells by modulating gap junction intercellular communication activity, we examined Panax notoginseng saponins-potentiated mineralization of these bone marrow stromal cells under osteogenic induction in the presence or absence of $18 \alpha$-lycyrrhetinic acid. A marked rise in alkaline phosphatase activity was observed

Li/Chang/Chen/Liu/Liu/Wang/Hou/Huang/Du 
in bone marrow stromal cells treated with $100 \mu \mathrm{g} / \mathrm{ml}$ Panax notoginseng saponins; this rise, however, was significantly attenuated by $18 \alpha$-glycyrrhetinic acid $(P<0.01)$ (Fig. 3G). Alizarin red S staining further revealed that $100 \mu \mathrm{g} / \mathrm{ml}$ Panax notoginseng saponins also significantly augmented the mineral content in bone marrow stromal cells under osteogenic induction, which, however, could be significantly attenuated by $18 \alpha$-glycyrrhetinic acid pretreatment (Fig. $3 \mathrm{H}$ and 3I) $(P<0.01)$. These findings demonstrated that Panax notoginseng saponins modulated gap junction intercellular communication activities in bone marrow stromal cells.

Panax notoginseng saponins stimulate osteogenesis of bone marrow stromal cells by targeting osteogenesis-associated genes

We further examined whether Panax notoginseng saponins potentiated the osteogenesis of bone marrow stromal cells by targeting genes associated with osteogenesis. Our RT-PCR analysis showed that, at day 14 of treatment, Panax notoginseng saponins 100 $\mu \mathrm{g} / \mathrm{ml}$ markedly increased the mRNA levels of alkaline phosphatase, core-binding factor a 1 , and bone sialoprotein (Fig. 4A and 4B). On the other hand, Panax notoginseng saponins caused a noticeable reduction in the transcript levels of PPAR $\gamma 2$. The above findings indicated that Panax notoginseng saponins stimulated the osteogenesis of bone marrow stromal cells by targeting genes associated with osteogenesis. We further treated bone marrow stromal cells with Panax notoginseng saponins in the presence or absence of $18 \alpha$-glycyrrhetinic acid. Our RT-PCR analysis showed that $100 \mu \mathrm{g} / \mathrm{ml}$ Panax notoginseng saponins noticeably increased the transcript levels of alkaline phosphatase, core-binding factor a1, and bone sialoprotein; this increase, however, was significantly attenuated by the presence of $18 \alpha$-glycyrrhetinic acid $(P<0.01)$ (Fig. $4 \mathrm{C}$ and $4 \mathrm{D})$. In addition, $100 \mu \mathrm{g} / \mathrm{ml}$ Panax notoginseng saponins markedly reduced the mRNA levels of PPAR $\gamma 2$, which, however, could be significantly lessened by $18 \alpha$-glycyrrhetinic acid $(P<0.01)$. These findings further demonstrated that Panax notoginseng saponins potentiated osteogenesis of bone marrow stromal cells by modulating the expression of osteogenesisassociated genes and that gap junction intercellular communication was involved in Panax notoginseng saponins-potentiated osteogenic differentiation of bone marrow stromal cells.

Promote Effects of Panax Notoginseng Saponins on Osteogenesis
Panax notoginseng saponins modulate the expression of RANKL and osteoprotegerin in bone marrow stromal cells under osteogenic induction through involvement of intercellular gap junctions

We further examined the effect of Panax notoginseng saponins on the expression of RANKL and osteoprotegerin, two proteins critically involved in osteoclastogenesis [21]. We measured the levels of RANKL and osteoprotegerin by ELISA and found that RANKL levels were reduced in bone marrow stromal cells upon osteogenic induction $(P<0.05)$ and this reduction was accentuated by the presence of $100 \mu \mathrm{g} /$ $\mathrm{ml}$ Panax notoginseng saponins $(P<0.01)$ (Fig. 5A). Additionally, bone marrow stromal cells under osteogenic induction exhibited a marked increase in the levels of osteoprotegerin $(P<0.05)$, which was further significantly augmented by $100 \mu \mathrm{g} / \mathrm{ml}$ Panax notoginseng saponins $(P<0.01)$ (Fig. 5B). Furthermore, 18 $\alpha$-glycyrrhetinic acid attenuated Panax notoginseng saponins-induced reduction in the levels of RANKL $(P<0.05)$ and Panax notoginseng saponins-mediated increase in the levels of osteoprotegerin $(P<0.01)$ (Fig. 5.A and 5B). In addition, bone marrow stromal cells under osteogenic induction showed a RANKL/osteoprotegerin ratio of 0.27 while those cells treated with $100 \mu \mathrm{g} / \mathrm{ml}$ Panax notoginseng saponins had a RANKL/osteoprotegerin ratio of 0.12(Fig. 5.C). Additionally, bone marrow stromal cells treated with both Panax notoginseng saponins and $18 \alpha$-glycyrrhetinic acid had a RANKL/osteoprotegerin ratio of 0.30 . These results indicated that Panax notoginseng saponins downregulated the expression of RANKL and at the same time upregulated the expression of osteoprotegerin in bone stromal cells under osteogenic induction with the involvement of intercellular gap junction communication.

\section{Discussion}

Bone remodeling underlies the process of bone repair or osteogenesis in many important bone diseases. Defective osteogenesis is a pivotal factor for the onset of osteoporosis, which is characterized by reduced bone mass and deteriorated bone microstructures with noticeably increased risk of bone fractures [22]. Defective osteoblast differentiation, among other causes, may also contribute to osteoporosis $[23,24]$. Bone marrow stromal cells are regarded as putative progenitors of osteoblasts 
in vivo and could be induced to differentiate into osteoblasts in vitro [25]. Promotion of proliferation and induction of differentiation of bone marrow stromal cells could offer a promising alternative therapeutic approach for osteoporosis and other bone diseases in which there are significant ongoing bone remodeling activities. Consistent with others, we also showed here that osteogenesis of primary rat bone marrow stromal cells could be effectively induced in vitro, demonstrating that these cells could be expanded in vitro to provide a ready source of early passage primary bone marrow stromal cells.

Panax notoginseng, a medicinal Chinese herb, has been used to treat bone fracture for over a thousand years and its major component, ginsenoside $\mathrm{Rg}_{1}$, was found to stimulate the proliferation of bone marrow stromal cells and increase the number of osteoblasts in vitro $[10,11]$. We also showed here that Panax notoginseng saponins could dose-dependently promote the proliferation and potentiate the osteogenesis of bone marrow stromal cells. However, the mechanisms whereby Panax notoginseng saponins promote osteogenesis of bone marrow stromal cells have hitherto remained undefined. Bone formation is a spatial cascading process, which initiates from the differentiation of bone marrow stromal cells into osteoprogenitor cells and then preosteoblasts and osteoblasts followed by matrix mineralization [26]. Gap junction intercellular communication has been shown to be required for normal osteoblastic differentiation and for bone repair[20]. We found here that increased gap junction intercellular communication activity was associated with osteogenic induction of bone marrow stromal cells in vitro, which was further enhanced by Panax notoginseng saponins, suggesting that Panax notoginseng saponins could stimulate osteogenesis of bone marrow stromal cells by modulating gap junction intercellular communication activity. This is further confirmed by the finding that $18 \alpha-$ glycyrrhetinic acid, a gap junction inhibitor, markedly attenuated Panax notoginseng saponins-mediated increase in gap junction intercellular communication activity in bone marrow stromal cells. These findings together shed light on a potential mechanism whereby Panax notoginseng saponins promote osteogenesis of bone marrow stromal cells.

Undifferentiated bone marrow stromal cells coexpress genes characteristic of several mesenchymal lineages including adipocytes and osteoblasts. Commitment of primitive pluripotential cells to specific lineages is marked by the activation of key transcription factors, which, in turn, turn on the expression of downstream tissue-specific genes [27]. We further examined whether Panax notoginseng saponins promoted osteogenesis of primary bone marrow stromal cells by modulating the expression of osteogenesis-associated genes such as the gene encoding core-binding factor a 1 , which is a master regulatory protein in bone marrow stromal cells and the predominant transcriptional activator of osteoblastassociated genes [28-30]. We found that Panax notoginseng saponins markedly increased the mRNA transcript levels of genes encoding proteins involved in osteogenesis such as alkaline phosphatase, core-binding factor a1, and bone sialoprotein while depressing the mRNA levels of PPAR $\gamma 2$, a key transcription factor that has been shown to inhibit osteogenesis [31]. Osteoblasts and adipocytes differentiate from a common precursor, the pluripotent mesenchymal stem cells in bone marrow and regulation of PPAR $\gamma 2$ activity has been shown to control the fate of these cells towards osteogenesis or adipogenesis. Suppression of PPAR $\gamma 2$ activity was associated with enhanced osteogenesis $[32,33]$. Our findings indicate that Panax notoginseng saponins not only stimulated the osteogenesis of bone marrow stromal cells but also tipped the balance of these cells away from adipogenesis towards osteogenesis. The effects of Panax notoginseng saponins on these osteogenesis-associated genes could be indirect through the modulation of gap junction intercellular communication activities. Changes in gap junction intercellular communication have been associated with changes in the expression of osteogenesisassociated genes in osteoblasts [34, 35]. Furthermore, Panax notoginseng saponins could suppress the expression of RANKL and augmented the increase in the levels of osteoprotegerin in bone marrow stromal cells under osteogenic induction. RANKL stimulates the maturation of preosteoclasts into functional osteoclasts by binding to RANK on preosteoclasts. Osteoprotegerin could function as a soluble decoy receptor and prevent RANKL from binding to RANK [26]. By changing the relative concentrations of RANKL and osteoprotegerin in bone, Panax notoginseng saponins changed the RANKL/osteoprotegerin ratio in favor of bone formation. We further showed that $18 \alpha$-glycyrrhetinic acid could partially reverse these actions of Panax notoginseng saponins, suggesting that these effects could be mediated by the modulation of gap junction intercellular communication activities by Panax notoginseng saponins.

In conclusion, we showed that Panax notoginseng saponins could potentiate the osteogenesis of primary bone marrow stromal cells in vitro by targeting genes

Li/Chang/Chen/Liu/Liu/Wang/Hou/Huang/Du 
encoding proteins involved in osteogenesis such as alkaline phosphatase, PPAR $\gamma 2$, RNAKL and osteoprotegerin. We further demonstrated that Panax notoginseng saponins may exert its osteogenesispotentiating effects by modulating gap junction intercellular communication activities. Our findings shed light on the mechanisms whereby Panax notoginseng, a medicinal herb that has been used to promote bone healing for over a thousand years, promotes bone formation. These findings indicate that Panax notoginseng saponins could be of value for treating diseases like osteoporosis in which there are significant ongoing bone remodeling activities.

\section{Acknowledgements}

This study was supported by the China Postdoctoral Science Foundation (No.20090450913), and the Administration of Traditional Chinese Medicine of Guangdong Province, China (No. 2008163 and No. 2010187).

\section{References}

1 Owen M, Friedenstein AJ: Stromal stem cells: marrow-derived osteogenic precursors. Ciba Found Symp 1988;136:42-60.

-2 Cancedda R, Mastrogiacomo M, Bianchi G, Derubeis A, Muraglia A, Quarto R: Bone marrow stromal cells and their use in regenerating bone. Novartis Found Symp 2003;249:133-43; discussion 437,70-4,239-41.

-3 Derubeis AR, Cancedda R: Bone marrow stromal cells (BMSCs) in bone engineering: limitations and recent advances. Ann Biomed Eng 2004;32:160165.

-4 Rodan GA: Introduction to bone biology. Bone 1992;13:S3-6.

-5 Stains JP, Civitelli R: Gap junctions in skeletal development and function. Biochim Biophys Acta 2005;1719:6981.

-6 Ng TB: Pharmacological activity of sanchi ginseng (Panax notoginseng). J Pharm Pharmacol 2006;58:1007-1019. Chen W, Dang Y, Zhu C: Simultaneous determination of three major bioactive saponins of Panax notoginseng using liquid chromatography-tandem mass spectrometry and a pharmacokinetic study. Chin Med 2010;5:12

8 Chen XC, Zhou YC, Chen Y, Zhu YG, Fang F, Chen LM: Ginsenoside Rgl reduces MPTP-induced substantia nigra neuron loss by suppressing oxidative stress. Acta Pharmacol Sin 2005;26:5662 .

-9 Rhule A, Navarro S, Smith JR, Shepherd DM: Panax notoginseng attenuates LPSinduced pro-inflammatory mediators in RAW264.7 cells. J Ethnopharmacol 2006;106:121-128.
Gong YS, Chen J, Zhang QZ, Zhang JT: Effect of 17beta-oestradiol and ginsenoside on osteoporosis in ovariectomised rats. J Asian Nat Prod Res 2006;8:649-656.

Lu XZ, Wang JH, Wu X, Zhou L, Wang L, Zhang XW, Cao KJ, Huang J: Ginsenoside Rg1 promotes bone marrow stromal cells proliferation via the activation of the estrogen receptormediated signaling pathway. Acta Pharmacol Sin 2008;29:1209-1214. Shen Y, Li YQ, Li SP, Ma L, Ding LJ, Ji $\mathrm{H}$ : Alleviation of ovariectomy-induced osteoporosis in rats by Panax notoginseng saponins. J Nat Med 2010;64:336-345.

Chang CH, Liao TC, Hsu YM, Fang HW, Chen CC, Lin FH: A poly(propylene fumarate)-calcium phosphate based angiogenic injectable bone cement for femoral head osteonecrosis. Biomaterials 2010;31:4048-4055.

Zhang YW, Morita I, Zhang L, Shao G, Yao XS, Murota S: Screening of antihypoxia/reoxygenation agents by an in vitro method. Part 2: Inhibition of tyrosine kinase activation prevented hypoxia/reoxygenation-induced injury in endothelial gap junctional intercellular communication. Planta Med 2000;66:119-123.

Zhang YW, Dou DQ, Zhang L, Chen YJ, Yao XS: Effects of ginsenosides from Panax ginseng on cell-to-cell communication function mediated by gap junctions. Planta Med 2001;67:417-422.

Fu L, Tang T, Miao Y, Zhang S, Qu Z, Dai K: Stimulation of osteogenic differentiation and inhibition of adipogenic differentiation in bone marrow stromal cells by alendronate via ERK and JNK activation. Bone 2008;43:40-47.
17 Kamijo M,Haraguchi T,Tonogi M,Yamane GY: The function of connexin 43 on the differentiation of rat bone marrow cells in culture. Biomed Res 2006;27:289-295.

Gregory CA, Gunn WG, Peister A, Prockop DJ: An Alizarin red-based assay of mineralization by adherent cells in culture: comparison with cetylpyridinium chloride extraction. Anal Biochem 2004;329:77-84.

$>19$ Yanagiya T, Tanabe A, Hotta K: Gapjunctional communication is required for mitotic clonal expansion during adipogenesis. Obesity (Silver Spring) 2007; 15:572-582.

20 Civitelli R: Cell-cell communication in the osteoblast/osteocyte lineage. Arch Biochem Biophys 2008;473:188-192.

21 Lacey DL, Timms E, Tan HL, Kelley MJ, Dunstan CR, Burgess T, Elliott R, Colombero A, Elliott G, Scully S, Hsu H, Sullivan J, Hawkins N, Davy E, Capparelli C, Eli A, Qian YX, Kaufman S, Sarosi I, Shalhoub V, Senaldi G, Guo J, Delaney J, Boyle WJ: Osteoprotegerin ligand is a cytokine that regulates osteoclast differentiation and activation. Cell 1998;93:165-176.

$>22$ Manolagas SC, Jilka RL: Bone marrow, cytokines, and bone remodeling. Emerging insights into the pathophysiology of osteoporosis. N Engl J Med 1995;332:305-311.

23 Diamond $\mathrm{T}$, Stiel D, Lunzer $\mathrm{M}$, Wilkinson M, Posen S: Ethanol reduces bone formation and may cause osteoporosis. Am J Med 1989;86:282288.

24 Rauch F, Travers R, Norman ME, Taylor A, Parfitt AM, Glorieux FH: The bone formation defect in idiopathic juvenile osteoporosis is surface-specific. Bone 2002;31:85-89 
25 Krause U, Harris S, Green A, Ylostalo J, Zeitouni S, Lee N, Gregory CA: Pharmaceutical modulation of canonical Wnt signaling in multipotent stromal cells for improved osteoinductive therapy. Proc Natl Acad Sci USA 2010;107:4147-4152.

26 Long MW: Osteogenesis and bonemarrow-derived cells. Blood Cells Mol Dis 2001;27:677-690.

27 Rasmussen TP: Embryonic stem cell differentiation: a chromatin perspective. Reprod Biol Endocrinol 2003;1:100.

28 Ducy P, Zhang R, Geoffroy V, Ridall AL, Karsenty G: Osf2/Cbfa 1: A transcriptional activator of osteoblast differentiation. Cell 1997;89:747-754.

29 Harada H, Tagashira S, Fujiwara M, Ogawa S, Katsumata T, Yamaguchi A, Komori T, Nakatsuka M: Cbfal isoforms exert functional differences in osteoblast differentiation. J Biol Chem 1999;274:6972-6978.
30 Karsenty G: Role of Cbfa1 in osteoblast differentiation and function. Semin Cell Dev Biol 2000;11:343-346.

1 Ichida F, Nishimura R, Hata K, Matsubara T, Ikeda F, Hisada K, Yatani H, Cao X, Komori T, Yamaguchi A, Yoneda T: Reciprocal roles of MSX2 in regulation of osteoblast and adipocyte differentiation. J Biol Chem 2004;279:34015-34022.

Akune T, Ohba S, Kamekura S, Yamaguchi M, Chung UI, Kubota N, Terauchi Y, Harada Y, Azuma Y, Nakamura K, Kadowaki T, Kawaguchi H: PPARgamma insufficiency enhances osteogenesis through osteoblast formation from bone marrow progenitors. J Clin Invest 2004;113:846855 .
Takada I, Suzawa M, Matsumoto K, Kato S: Suppression of PPAR transactivation switches cell fate of bone marrow stem cells from adipocytes into osteoblasts. Ann N Y Acad Sci 2007;1116:182-195. Lecanda F, Towler DA, Ziambaras K, Cheng SL, Koval M, Steinberg TH, Civitelli R: Gap junctional communication modulates gene expression in osteoblastic cells. Mol Biol Cell 1998;9:22492258.

Li Z, Zhou Z, Yellowley CE, Donahue HJ: Inhibiting gap junctional intercellular communication alters expression of differentiation markers in osteoblastic cells. Bone 1999;25:661-666. 